\title{
How sublexical association strength modulates updating: Cognitive and strategic effects
}

\author{
Caterina Artuso ${ }^{1}$ - Paola Palladino ${ }^{1}$
}

Published online: 18 October 2017

(C) Psychonomic Society, Inc. 2017

\begin{abstract}
In the current study, we investigated updating of long-term memory (LTM) associations. Specifically, we examined sublexical associations by manipulating preexisting LTM relations between consonant couplets (in encoding and updating phases), and explicitly instructed participants to engage with a specific strategy for approaching the task (item disjunction, grouping, or none). In two experiments, we used a multistep subject-based memory updating task in which we measured processing response times (RTs; Exp. 1, Exp. 2) and recognition RTs (Exp. 2). For the first time, in both experiments, we found costs in dismantling strong pre-existing associations from LTM and benefits in recreating strong preexisting associations. In addition, we found that control of irrelevant information was more difficult when this belonged to a strong association. Regarding task strategies, we showed that inducing a disjunction strategy enhanced updating, no matter the strength of the association. Results were discussed in the light of updating as a process of dismantling and recreating associations. The role of a specific strategic approach in enhancing the updating was also discussed.
\end{abstract}

Keywords Updating $\cdot$ Long-term memory $\cdot$ Working memory $\cdot$ Sublexical representation $\cdot$ Memory strategy

Working memory (WM) is a capacity-limited system, able to actively maintain sets of representations that are useful in specific cognitive tasks such as reading, math, or text comprehension (e.g., Daneman \& Carpenter, 1980; Palladino, Cornoldi,

Caterina Artuso

caterina.artuso@unipv.it

1 University of Pavia, Pavia, Italy
De Beni, \& Pazzaglia, 2001). Given capacity limits and the continuous flow of information needing to be processed, it is important to postulate the existence of a mechanism that allows WM content to be constantly updated.

Once selected, goal-relevant information is maintained actively and updated in WM, and subsequently modified when no longer relevant (Morris \& Jones, 1990). More specifically, updating could be described as a three-components process: retrieval, transformation, and substitution (see Ecker, Lewandowsky, Oberauer, \& Chee, 2010). Retrieval allows access to information in WM, within attentional focus. Transformation allows modification of a representation already held in WM, and substitution enables removal of previous (no longer relevant) items, replacing them with new information. Item substitution appears to be the most distinctive processing component of updating. Indeed, it differentiates updating from other WM tasks, such as recall, that do not involve single item substitution (see Ecker et al., 2010).

The basic definition of updating - that is, maintaining relevant information and inhibiting no-longer-relevant information - is usually applied to memory contents (see seminal work by Morris \& Jones, 1990). However, typically, achievement of an updating task is based on binding and unbinding processes between memory contents or, in addition, actualization of bindings between contents. Through a latent variable analysis, Schmiedek, Hildebrandt, Lövdén, Wilhelm, and Lindenberger (2009) found that updating (measured with an $n$-back task) and complex span tasks were highly correlated. This indicated that updating tasks measure WM as equally effectively as complex span tasks and potentially share common processing mechanisms of continuously building, maintaining, updating, and releasing (usually arbitrary) bindings between items. In a response-time (RT) based updating task, Artuso and Palladino (2014) reported that binding updating (vs. content updating) is a more sensitive process measure in 
explaining performance in classical (i.e., accuracy-based) updating tasks. Thus, the specific challenge in updating activity seems to be the monitoring of contextual bindings between contents. In the same vein, Boujut and Clarys (2015) supported the role of content-context bindings in retrieval of episodic memories (rather than single contents).

To date, no studies have specifically investigated this process of creation, maintenance, updating, and releasing of bindings (or associations) between elements that are not arbitrary but that are strengthened on the basis of long-term memory (LTM) organization. Indeed, an important and relatively neglected issue in updating research concerns how information retained in LTM can be updated differentially in ongoing processing.

Within this research debate, the numerical domain is also relevant. Lendínez, Pelegrina, and Lechuga (2011, 2014) found evidence of a similarity effect: numerical similarity produces facilitation during updating of information. When the numbers involved in updating were more similar (i.e., in numerical distance, or through sharing a digit), substitution occurred more quickly. The authors proposed that updating might be easier if the number presented is closer to the number stored in LTM. For example, two items that are stored in WM can overlap, as long as their representation share certain features (see, e.g., Nairne, 1990). Given that sets of features overlap to a various extent, the more similar the items, the greater the degree of overlap. When it is necessary to update a number that shares many features with another stored in memory, the process can be performed more quickly; fewer features of the second number need activation because shared features are already activated. As such, Lendínez et al. (2011, 2014) showed that updating RTs were faster when the distance between the number stored in memory and the number replacing it was smaller than when this distance was large. Indeed, they showed that the relation (based on numerical similarity) between old and new information involved in updating has an effect on RT.

\section{Lexical and sublexical associations in working memory}

The effects of LTM organization of information on WM performance can be observed at different processing levels, such as lexical and sublexical. Below, we will briefly review the literature relative to lexical and sublexical effects.

At the lexical level, Hulme, Stuart, Brown, and Morin (2003) studied the effect of stimulus (i.e., word) variables on memory performance. They found that high-frequency words (vs. low) elicit better recall (also known as "word-frequency effect"); this was accounted for by recall of high-frequency words benefitting from stronger preexisting interword associations in the experimental context. Similarly, Poirier and
Saint-Aubin (2005) showed that high-frequency words (similar to highly familiar words) are better recalled by virtue of their stronger long-term associative links (see also Thorn \& Frankish, 2005).

Similarly, at the sublexical level, Gathercole, Frankish, Pickering, and Peaker (1999) found that recall accuracy is greater for words containing high (vs. low) frequency phoneme combinations in English, also known as the "phonotactic effect." Here, the authors hypothesized that LTM knowledge is often integrated with temporary maintenance of information in WM. Performance would benefit from use of stored phonotactic representations for familiar words to fill in incomplete traces prior to output. In contrast, for nonwords (or unfamiliar words), no stored representations are available to reconstruct partial traces, and this, in turn, leads to diminished accuracy at recall. Moreover, recall is better for phonotactic frequencies of the language in WM and is specific for phoneme combination distribution within each language. In fact, the phonotactic effect elicits better recall for consonant-vowel-consonant nonwords containing consonant-vowel and vowel-consonant combinations, common in the language's native phonology, than for nonwords containing lowprobability consonant-vowel combinations. Accordingly, such effects would reflect influence of phonotactic (or sublexical) knowledge about properties of that language (see Gathercole et al., 1999). Similarly, Majerus, Van der Linden, Mulder, Meulemans, and Peters (2004) demonstrated that verbal WM is influenced by sublexical phonological knowledge; indeed, legal nonword repetition was significantly higher than that for illegal nonwords.

To account for LTM effects on recall performance, different explanations have been proposed, such as redintegrative, language-based, or probabilistic accounts. Redintegration of an item (i.e., reconstruction of an item) would operate effectively on high-frequency words because their phonological representations are more easily accessed by partial information. Accordingly, effects of item frequency on recall influence the relevant item only, and occur at the time the individual item is retrieved and recalled (see Gathercole et al., 1999; Hulme et al., 2003; Saint-Aubin, Ouellette, \& Poirier, 2005).

Majerus (2013) proposed an integrative framework of WM language-based accounts, where WM is an emergent property resulting from temporary activation of LTM knowledge in the attentional, linguistic, and executive processes network (see also Acheson \& MacDonald, 2009; Martin \& Saffran, 1992). Thus, temporary storage would result from recruitment of language, attentional, and serial order processing systems. Other contributions also underline interaction between LTM and STM by using incidental statistical learning and probabilistic models of learning (see Majerus, Perez, \& Oberauer, 2012).

In sum, a general achievement for memory studies on both words/nonwords and phoneme combinations (i.e., sublexical units) seems to be the strong and direct interaction between 
verbal WM processing and language LTM networks. Indeed, all accounts presented above aim to explain the common and consistent finding that high-frequency words/phonemes tend to enhance memory recall.

\section{Strategic effects in working memory}

Strategic effects in WM performance have been widely demonstrated (see, e.g., Chein \& Morrison, 2010; McNamara \& Scott, 2001; Morrison \& Chein, 2011; Morrison, Rosenbaum, Fair, \& Chein, 2016). In particular, performance is improved and boosted by use of efficient strategies. For example, McNamara and Scott (2001) showed that when readers were instructed and trained to use a chaining strategy (i.e., a mnemonic strategy to create semantic associations between words in a list), they recalled a greater number of words after training compared to those untrained or those trained with less effective strategies (e.g., a retrieval strategy). In turn, trained readers are more able (and more inclined) to make inferences, when needed.

It seems also that a strategic approach to a typical WM task produces a beneficial effect on performance. Morrison et al. (2016) assessed the use of strategies across a number of WM tasks including both simple (e.g., free recall or immediate serial recall) and complex tasks (e.g., running memory or operation span task). They reported that spontaneous strategy use (such as rehearsal, grouping, imagery, checklist) is heterogeneous across different tasks, and that task performance did not vary as a function of strategy use.

As previously noted, updating is a complex WM task where participants are likely to use some form of strategy to engage with it. Very few studies, however, have investigated the role of strategy in a classical updating task, that is, accuracy-based ones, such as Morris and Jones's running memory task (but see Bunting, Cowan, \& Saults, 2006; Palladino \& Jarrold, 2008). Here, the participant has to listen to differing-length lists of letters and then is asked to recall a fixed number of the last items of the list (e.g., the last three). However, a possible bias with this task arises from the use of passive recency-based strategies. The participant does not usually process each incoming item via updating of its activation but listens passively until the list ends, and tries to recall the most recently presented items (see Palladino \& Jarrold, 2008).

Usually, these strategies are investigated a posteriori, once the task has been completed. To our knowledge, no studies have manipulated different strategy use directly, investigating how these may impact performance in an updating task. As such, it would be of interest to fill this gap in the literature, by instructing participants to use a specific strategy in performing the task.

\section{The current study}

Previous studies have mainly focused on the simple task of recall, which entails temporary maintenance of information in WM (see also Cowan, 2017). Here, we focused instead on the interaction between LTM and updating, a complex WM function that comprises not only temporary maintenance of information but also item substitution (see Ecker et al., 2010). To our knowledge, only one study has investigated WM updating of LTM associations between verbal materials (see Artuso \& Palladino, 2016). Here, the authors manipulated the strength of the association between stimuli from LTM, which might produce differences in the updating process itself. Following literature considering beneficial effects of highly associated LTM information (based on lexical and sublexical frequencies; see Gathercole et al., 1999; Hulme et al., 2003), the authors investigated whether strong or weak associations were updated differently. Strength was represented by the frequency of sublexical associations between consonants in the spoken language. Association strength was manipulated at encoding, in order to observe how strong and weak associations were subsequently updated in an ongoing task. Overall, strong associations at encoding became weak at updating. In fact, it was shown that the stronger the association was, the higher the interference with the updating process was (i.e., longer RTs to substitute information and to control for irrelevant information). Thus, a processing cost was found for updating, showing the reverse effect to recall, which is boosted by strength of the association.

The purpose of the present study was to investigate further how specific sublexical associations from LTM are updated in WM. Here, we extended previous findings in two ways. First, we manipulated the strength of associations at both encoding and updating; second, considering the importance of a strategic approach in complex tasks, we aimed to investigate how participants engage in the task, once instructed to use a specific strategy.

To this end, we administered an updating task to our sample that minimized cognitive costs (i.e., the task was subjectpaced). The subject-paced measure is more ecologically valid and minimizes the impact of individual differences in strategy use (see Morrison et al., 2016). In addition, it enables separation of each individual process, and separate measurement of each specific phase of encoding and updating. Moreover, in previous studies with verbal and nonverbal materials (see e.g., Artuso \& Palladino, 2014; Artuso, Palladino, \& Ricciardelli, 2015), significant individual differences were not observed between slow and fast responding participants, but all performed within the average processing rate.

We formulated three main hypotheses, considering the relation between two items maintained at the same time in WM. Therefore, our main focus was not on possible shared features but on processes of dismantling and recreating (via item- 
substitution) associations between items. With these two crucial steps in mind, we aimed to investigate the potential role of both to-be-dismantled associations and those to be recreated. Overall, we expect that strong associations should be updated with weak ones, with greater difficulty (i.e., longer RTs). Here, a stable preexisting association from LTM has to be broken, and this should produce a cost. Conversely, we expect that weak associations should be updated with strong with less difficulty (i.e., shorter RTs). In other words, a preexisting LTM association has to be recreated, and this should produce an advantage. This finding would provide original evidence on how different associations' strength affect updating.

Typically, the use of strategies in WM updating has not been manipulated directly but assessed after task completion (see, e.g., Bunting et al., 2006). However, considering the impact strategies have on WM performance in general (e.g., Morrison et al., 2016), we believe it is of interest to understand how participants are strategic in engaging with the updating task and to observe which strategy is most effective for task performance. Overall, updating requires flexibility (i.e., an ability to create and dismantle item-set configurations rapidly), but the nature of the material (LTM sublexical associations) favors creation of stable associations. Here, we chose to instruct participants explicitly, suggesting engagement in a predetermined strategy that could favor the associative nature of the material, or the reverse. Before starting the task, we instructed our participants verbally, requesting the use of a specific strategy (i.e., either to separate the items or to group them). Following the three-components model of Ecker et al. (2010), where item substitution represents the distinctive component of updating, we predict that participants who use a disjunctive strategy will update their memory set more efficiently, as they should substitute the to-be-updated item more quickly (shorter RTs). In fact, if they treat the items individually (at encoding), we predict that subsequent single item substitution (i.e., at updating) should be more efficient. Instead, when they will use an item-grouping strategy, subsequent substitution should be disadvantaged.

Finally, we predict that instruction could affect the strength of association in two possible ways. First, we anticipate that instruction will not interact with strength. If so, the disjunction strategy should improve updating. Thus, when encoding information, participants may create less stable associations (where the items are treated as single units), and after, at updating, it should be easier to break those associations whatever their strength (see also the previous paragraph). Alternatively, we could anticipate modulation in accordance with association strength. Accordingly, a disjunction strategy should be more beneficial for strong associations to be updated with weak. In other words, if associated items are treated individually, this should mainly affect strong associations, where items are well connected with each other and more difficult to separate (as they benefit from preexisting LTM association). The disjunction strategy should therefore reduce RTs when updating, particularly in respect of strongly associated units rather than weakly associated ones. Weak associations should necessitate disjunction less, as items are already unbound (i.e., they do not take advantage of any preexisting LTM association).

\section{Experiment 1}

Following evidence that WM performance is effectively and directly linked to stored LTM representation (see, e.g., Gathercole et al., 1999), we created both strong and weak sublexical associations, based on the frequency of cooccurrences of sublexical units in the spoken Italian language. In order to understand the updating process more clearly and control the whole processing sequence effectively, here, the strength of letters' LTM association was manipulated, both when participants encoded the information and in subsequent updating of that information. Therefore, the updating process sequence was manipulated to create three conditions: (1) a condition with strong associations, to be updated with weak ones; (2) a condition with weak associations, to be updated with strong ones; and (3) a condition with weak associations to be updated with other weak associations.

This manipulation was devised in order to test our hypotheses regarding the role of LTM in the updating process as innovatively and extensively as possible. The task required participants, once each set of three units (i.e., well within average span) of information was encoded, to update this set; namely, to change part of their memory representation by partially substituting one item with a new one.

We hypothesized longer RTs for strong associations, when updated with weak associations (i.e., a cost to dismantle a preexisting association from LTM), and conversely, shorter RTs for weak associations, when updated with strong ones (i.e., a benefit when a preexisting association is recreated). Any finding showing that strong associations are more resource demanding (i.e., when they are to be updated with weak associations) would then support previous findings. In addition, by demonstrating the opposite finding (i.e., that weak associations are more easily updated with strong ones), we would add compelling evidence regarding the role of association strength on updating. In this vein, we expect that weak-to-weak conditions (i.e., weak association to dismantle, and weak to recreate) will show shorter RTs than strong-to-weak conditions (here, a strong association has to be dismantled, and therefore this should produce a cost, by virtue of preexisting LTM association). Weak-to-weak conditions are predicted to show longer RTs that weak-to-strong conditions (a strong association has to be recreated, and 
Table 1 Overview of different associations and predictions for associations and predictions for Experiment 1 and Experiment 2

\begin{tabular}{llll}
\hline Association to dismantle & Expected results & Association to recreate & Expected results \\
\hline Strong & $\begin{array}{c}\text { Cost in dismantling a strong preexisting } \\
\text { association (longer RT) } \\
\text { Cost in dismantling a strong preexisting } \\
\text { association (longer RT) } \\
\text { Strong }\end{array}$ & Weak & $\begin{array}{c}\text { Cost in recreating a weak association } \\
\text { (longer RT) } \\
\text { Benefit in recreating a strong preexisting } \\
\text { association (shorter RT) }\end{array}$ \\
Weak & Benefit in dismantling a weak association (shorter RT) & $\begin{array}{c}\text { Strong } \\
\text { Cost in recreating a weak association } \\
\text { (longer RT) }\end{array}$ \\
Benefit in recreating a strong preexisting \\
association (shorter RT)
\end{tabular}

therefore LTM strength should produce an advantage). See Table 1 for a summary.

In essence, the comparison between conditions will show if the updating process works in all conditions through dismantling bindings and building new ones, and whether this is affected similarly by each strength of association. In addition, we anticipate that the disjunction strategy could favor updating by starting a process of breaking links between items and weakening associations.

\section{Method}

\section{Participants}

A sample of 90 undergraduates students from the local university (mean age $=21.92$ years, $S D=2.35,28$ males) participated in exchange for course credit, in a session of around 40 minutes. Informed consent was obtained. Each participant was randomly assigned to one of three instruction groups (30 per group).

\section{Stimuli}

The stimuli were sublexical units between two consonants of the Latin alphabet. The association was based on LTM representation of consonants; that is, on the basis of their combined frequency in the spoken Italian language. We started from the lexicon of frequency of Italian spoken language (see De Mauro, Mancini, Vedovelli, \& Voghera, 1993); this is a corpus of about 490,000 words collected in four main Italian cities, emerging from different subgroups of discourse. We then derived strong and weak phonotactic associations between consonants, based on the sublexical unit letters frequency. Strong associations are those derived from sublexical units with at least three occurrences in the corpus; weak associations are those with less than three occurrences in the corpus. For example, if "TRO" was a high-frequency sublexical unit, we derived the association "T-R."

We employed the following set of consonants: B C D F G H L N P R S T. Strong associations were: TR, SP, PR, NT, BR,
CH, GR, FR. Weak ones were: FL, SN, GH, PS, GL, RD, ND, LT. Strong and weak associations between consonants were controlled in order to avoid obviously familiar or meaningful couplets. Each association was possible at the sublexical level between consonants of the Italian language.

In order to avoid ceiling (i.e., with two items) or floor effects (i.e., with four items), we used memory sets composed of three letters (i.e., trigrams), which have been established as being within average memory span (Cowan, 2001). Some letters were overrepresented relative to others, but we controlled for this bias by randomizing them across association strengths. Further, the position of the sublexical unit within the trigram (i.e., in Positions 1-2 or 2-3) was randomized between trials.

The third letter of each trigram was another consonant, which was always unrelated with the other two. Specifically, the link between the sublexical unit and the third letter was always linguistically impossible in Italian (e.g., see the example from Fig. 1, where $\mathrm{CH}$ is a strong association and the link between $\mathrm{H}$ and $\mathrm{B}[\mathrm{HB}]$ is impossible in Italian). This was done in order to avoid the third letter being associated in either a strong or weak manner, or in some other meaningful way.

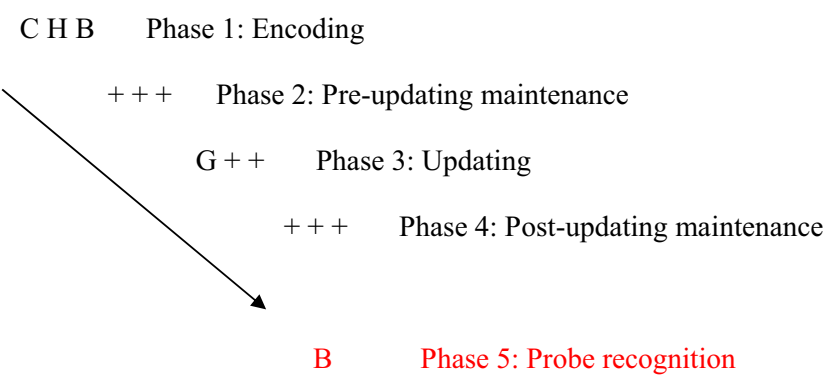

Fig. 1 An example of an experimental trial presenting a strong association updated with a weak association. Phase 1: After encoding the first trigram (i.e., CHB), participants had to maintain it actively in memory (Phase 2). At Phase 3, they were instructed to update the association by dismantling the association (i.e., here, between $\mathrm{C}$ and $\mathrm{H}$ ) and substituting it with another (i.e., $\mathrm{G}$ and $\mathrm{H}$ ). This presented the new trigram (i.e., GHB). Phase 4: Participants were asked to maintain this last trigram. Finally (Phase 5), a single probe was displayed. Here, they had to recognize if the probed consonant belonged or not to the most recent trigram they held in mind. In this example, a positive probe was displayed 


\section{Design and analyses}

A design with three factors was implemented: Strength and phase were within-participants factors, and instruction was between participants. The variable strength had three levels: strong-to-weak, weak-to-strong, and weak-to-weak associations. Strong-to-weak associations represented associations between letters that was strong at encoding, but once updated was modified with a weak association, such as from GR (strong) to GH (weak).

Weak-to-strong associations represented associations between letters that was weak at encoding and modified into a strong association on updating (e.g., GH to GR). Finally, weakto-weak associations represent associations between letters that occurred when the association was weak at encoding and updated with another weak association (such as GL to FL).

Each trial had four phases: (1) encoding (i.e., to study/ encode the initial trigram); (2) maintenance preupdating (i.e., to maintain the encoded trigram; three pluses); (3) updating (i.e., partial substitution into the trigram); and (4) maintenance post updating (i.e., maintenance of the modified trigram; three pluses; see also Procedure section).

For consistency with previous studies (e.g., Artuso \& Palladino, 2011), we used two maintenance phases, mainly to give a clear structure to the task and to characterize and measure the updating phase effectively. These phases were represented on-screen by a series of three pluses (to favor active memory maintenance of previously studied information).

The instruction factor had three levels: disjunction, grouping, or no strategy. For disjunction, the participant had to memorize the item (each single letter separately); that is, to consider each letter as a separate element distinguished from adjacent ones and not as a whole element (e.g., the trigram CHB was to be coded as C, separately from $\mathrm{H}$ and B). For grouping, the participant had to consider each element as if it was a whole set of elements. For the no-strategy level, the participants were free to use the strategy they preferred.

In addition, to make the task less predictable and ensure participants were engaged, we included some control trials (about 20\%). Here, no updating occurred, and only maintenance was needed throughout the trials. These were not included in further analyses but were checked to ensure that all updating trials had longer RTs than control ones overall $(p<$ .05 for each comparison, i.e., control vs. strong-to-weak, vs. weak-to-strong, and weak-to-weak).

\section{Procedure}

The task was administered on a standard PC and was constructed by adapting experimental procedure from previous studies (see Artuso, Cavallini, Bottiroli, \& Palladino, 2017; Artuso \& Palladino, 2011, 2016). It consisted of four-phase subject-paced trials where participants pressed the spacebar to start each trial, and after each phase, in order to proceed with the task.

In each phase, trigrams were always displayed in the center of the screen. Each trial started with an encoding phase (Phase 1; see Fig. 1, where a strong-to-weak association is represented), where participants had to memorize the first trigram of consonants (e.g., C H B). A preupdating maintenance phase followed (Phase 2), where three pluses were displayed; this indicated that the previously encoded trigram had to be actively maintained. Then, at the updating phase (Phase 3), the participant had to substitute the no-longer-relevant information (here, C) with newly and relevant information (here, G). Concurrently, they needed to maintain previously relevant detail (here, HB), thus updating the trigram (here, from CHB to GHB). Finally, a postupdating maintenance (Phase 4) ended the sequence, to control for recency biases (see also Fig. 1).

Only one letter of the trigram had to be updated; this letter could be presented in any position of the trigram (i.e., left letter, right letter, or center). Position was balanced across trials, and only new consonants were presented across each phase. When a consonant did not change, a plus symbol was presented in order to encourage active maintenance of previously encoded/memorized information.

At the end of each trial (Phase 5), participants were presented with a probe-recognition task: a single red consonant was displayed in the center of the screen. Here, they had to indicate whether this belonged to the most recently studied trigram or not. They responded by pressing one of two keys on the keyboard; one ( $M$ for Yes) for probes requiring a positive answer (i.e., belonging to the final trigram of the trial); another one ( $Z$ for No) for probes requiring a negative answer (i.e., not previously presented in the trial. For these, we included both intrusion (i.e., probes encoded and them substituted at updating) and negative probes (i.e., probes not presented in that trial), mixed within the trial. Participants were randomly assigned to one of the three instruction groups and tested individually. Each participant was given standard instructions (i.e., background to the task and encouragement to be as fast, but accurate, as possible), except for the final sentence that was varied across groups. The disjunction strategy group was told, "Try to encode/remember each letter separately from others as distinct elements throughout the trial." The grouping strategy group was told, "Try to consider each letter as joined to the others adjacent as related to the others and to code/remember each letter as a whole, not separately." The no-strategy group was simply told to perform at their best and get as many trials correct as possible.

Afterward, each participant was presented with a practice block of eight trials to familiarize with the task. Two hundred and twenty-eight trials were then presented, shared equally in four blocks. We recorded subjectpaced RT at each of the four phases as well as proberecognition accuracy at Phase 5. 


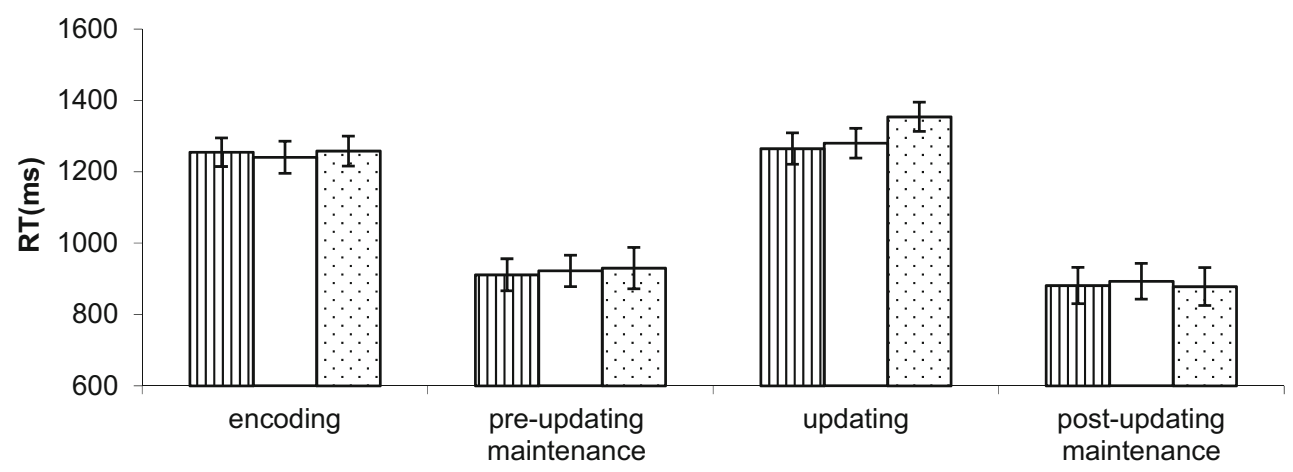

Fig. 2 Experiment 1. Mean RTs for each association strength as a function of phase

\section{Results}

\section{Accuracy analysis and data treatment}

Participants performed accurately in an average of $95 \%$ of trials. We ran an ANOVA, with strength (weak-toweak, weak-to-strong, strong-to-weak) as a withinparticipants factor, and instruction (disjunction, grouping, no strategy) as a between-participants factor, on mean accuracy rates of positive and negative responses No effect reached significance $(p>.05)$. Only subject-paced RTs for trials that ended with correct probe recognition were analyzed. Trials with RTs of less than $150 \mathrm{~ms}$, or exceeding a participant's mean RT for each condition by more than three intraindividual standard deviations, were considered outliers and therefore excluded from further analyses $(3.40 \%)$.

\section{RT analyses}

An ANOVA with strength (weak-to-weak, weak-to-strong, strong-to-weak) and phase (encoding, preupdating maintenance, updating, postupdating maintenance) as withinparticipants factors, and instruction (disjunction, grouping, no strategy) as a between-participants factor, was conducted on subject-paced processing RTs. Below, we report all statistics for main effects and interactions.

The main effect of instruction did not reach significance ( $p$ $>.05)$; neither did the interaction between instruction and strength $(p>.05)$. Results showed a significant main effect for strength, $F(2,86)=24.62, \eta_{\mathrm{p}}{ }^{2}=.36, p<.001$, with longer RTs on average for the strong-to-weak associations compared to the other two types. In addition, a main effect of phase reached significance, $F(3,85)=65.05, \eta_{\mathrm{p}}{ }^{2}=.70, p<.001$, showing longer RTs for both encoding and updating compared to the two maintenance phases.

The two-way interactions between phase and strength reached significance, $F(6,82)=6.24, \eta_{\mathrm{p}}{ }^{2}=.31, p<.001$.
Moreover, the interaction between phase and instruction also achieved significance, $F(6,172)=2.88, \eta_{\mathrm{p}}{ }^{2}=.091, p=.011$ (see further analyses below). The three-way interaction was not significant $(F<1)$.

\section{Phase by strength}

Planned comparisons, using Tukey's method, showed that at encoding, there were no significant differences between trials $(p>.05)$, as shown in Fig. 2. Similarly, there were no significant differences in maintenance phases, before or after updating $(p>.05)$.

At the updating phase, we found longer RTs for strong associations to be updated with weak ones $(1,354 \mathrm{~ms})$, relative to both weak associations to be updated with strong (1,280 $\mathrm{ms})$ and to weak associations updated with weak (1,265 ms). The difference between these latter two association types was not significant $(p>.05)$.

\section{Instruction by phase}

Planned comparisons, using Tukey's method showed that, at encoding, the disjunction instruction led to shorter RTs, compared to both grouping and no strategy. Grouping and no strategy did not differ $(p>.05)$. Similarly, for all instruction conditions, no differences were observed at maintenance $(p>$ .05). Similarly, at updating, the disjunction instruction lead to shorter RTs (1,228 ms) compared to both grouping (1,362 ms) and no strategy $(1,337 \mathrm{~ms} ; p<.05)$.

Therefore, we showed the disjunction instruction was effective in the updating phase, producing shorter RTs compared to the other two strategies as shown in Fig. 3. We found no interaction between strength of association and instruction, indicating that instructed strategies affected the updating process similarly, regardless of the strength of associated sublexical units. 


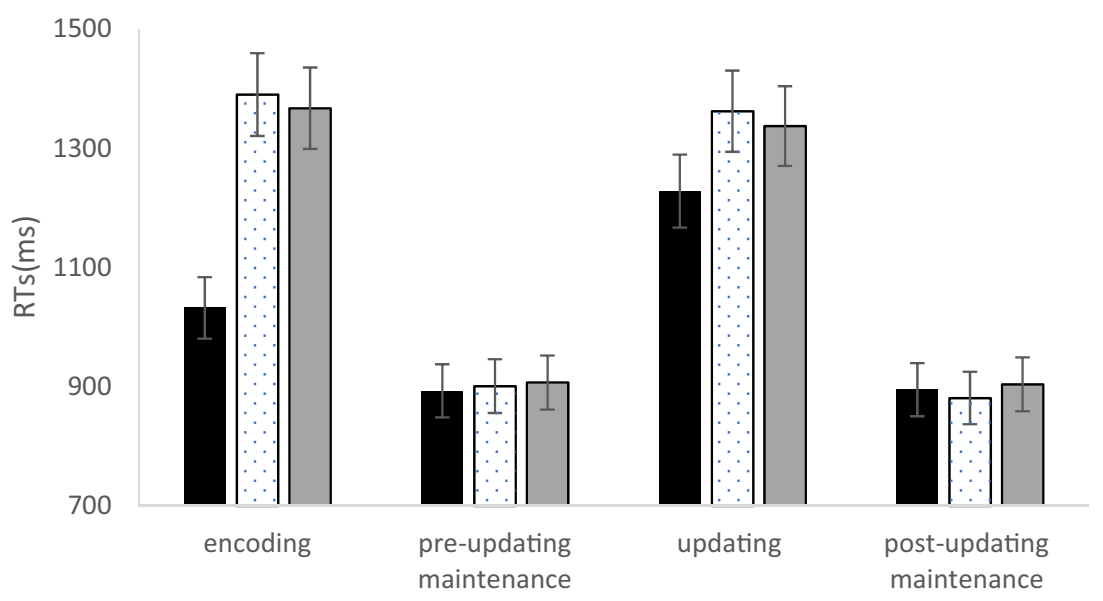

Fig. 3 Experiment 1: Mean RTs for each phase as a function of instruction

\section{Discussion}

We demonstrated that a strong association needs more time to be disconnected from a weak association. In addition, we found the opposite result-weak associations needed shorter latency to be updated with strong ones. In other words, there was cost in dismantling a strong preexisting association and, simultaneously, a benefit in recreating a strong preexisting association. However, we did not show that weak-to-weak associations had longer latencies than weak-to-strong associations; in fact, their difference did not reach significance.

In addition, we found that induction of a disjunction strategy interacted with the task positively. When participants use the disjunction strategy, they both encoded and updated the information faster regarding such encoding advantage (i.e., using the disjunction strategy); we believe this represents a direct effect of our manipulation. Participants were explicitly instructed to use a strategy of keeping the items disjointed; this is reflected in their response latencies and, in turn, confirms the efficacy of our manipulation.

The originality of our result is in the finding of an effect on the updating phase. More specifically, we observed that when participants were instructed to use the disjunction strategy, this facilitated the updating process (in terms of a reduction in RT) no matter which association had to be updated. According to our hypotheses, when participants keep items separated (working against spontaneous/natural binding), they were faster in dismantling associations and substituting irrelevant items with new ones (or, alternatively, they were faster in updating information). This was not the case when another strategy (i.e., grouping) was applied.

\section{Rationale and hypotheses for Experiment 2}

In order to warrant replication of results and to clarify aspects of our design, we have undertaken a second experiment, controlling for several potential biases in Experiment 1. As the two maintenance phases in the task do not have particular theoretical importance (i.e., have been included for consistency only), we have included these in the design but not in the analyses for Experiment 2.

Furthermore, considering that both the grouping and nostrategy instructions elicited similar RTs, here, we administered two different types of instructions to our participants (i.e., grouping and disjunction) in order to examine two experimental groups with better focused analyses. Moreover, in Experiment 2, we added a fourth condition; a strong association updated with another strong association. In addition to the hypotheses formulated for Experiment 1, we predicted that the strong-to-strong condition should be less difficult (shorter RTs) than the strong-to-weak condition. In this instance whereas in the former case a strong association has to be recreated (and this usually produces a benefit- shorter RTs), in the latter, a weak association is recreated and typically, this produces a cost (longer RTs). Conversely, we predicted that when strong associations are updated with either weak or strong associations, they will require longer RTs, as in both cases the to-be-dismantled association is strong, and this should always produce a cost. In addition, adding a fourth condition allowed us to explore the relative importance of costs/benefits in dismantling and recreating associations of differing strength (see Table 1).

In addition, in Experiment 1 we considered processing and not recognition RTs, thus neglecting to analyze possible interference control effects. For this reason, in Experiment 2, we focused on both processing and recognition RTs, distinguishing and analyzing accuracy and RT for all three probe types (positive, intrusion, and negative). The index of $\mathrm{RT}$ is more sensitive in detection of potential processing costs (see, e.g., Artuso \& Palladino, 2014). Thus, in addition to RTs that track the updating process, we measured costs of controlling for interference via probe recognition RTs. 
Indeed, we anticipated that stronger associations are also more active in LTM and thus are likely to be more interfering and difficult to control. Moreover, we also focused on recognition RTs in accordance with previous findings that showed an effect of association strength on recognition (see Artuso \& Palladino, 2016).

Finally, in this experiment, we did not randomize the position of the associated couplet within the trigram but controlled and analyzed the position of the sublexical unit within the trigram. This could be initial position $(1,2)$ or final $(2,3)$. It could be particularly important to control for strong sublexical units, as position might be influential. For example, strong associations in the initial position $(1,2)$ might be especially strong (e.g., even slower to convert from strong to weak, or even faster to convert from weak to strong).

\section{Experiment 2}

\section{Method}

\section{Participants}

A sample of 50 undergraduates students from the local university (mean age $=21$ years, $S D=1.01,21$ males) participated in exchange for course credit, in a session that lasted about 40 minutes. Informed consent was obtained. Each participant was randomly assigned to one of two instruction groups (25 per group).

\section{Stimuli, design, and procedures}

The stimuli were the same as in Experiment 1. In addition, we created strong-to-strong sublexical units. A design with four factors was implemented: strength, phase, and position were within-participants factors; instruction was between participants. The variable strength had four levels: strong-to-weak, weak-to-strong, weak-to-weak, and strong-to-strong. Strongto-strong represented an association where the encoded strong sublexical unit was subsequently updated with a strong couplet (e.g., TR to PR).

Position had two levels: the sublexical unit could be placed at the beginning of the trigram (1-2) or at the end (2-3). Due to constraints of the Italian language, for the strong-to-strong association, the sublexical unit could be only in position 1-2 and never in position 2-3. Thus, the design is not fully randomized.

As in Experiment 1, each trial had four phases, but only encoding (Phase 1; see Fig. 1) updating (Phase 3), and probe recognition (Phase 5) were analyzed. The instruction factor had two levels: disjunction or grouping.

As in Experiment 1, to make the task less predictable and ensure participants' engagement, we included control trials (about 20\%). Here, no updating occurred, and only maintenance was required throughout the trial. These trials were not included in further analyses but were checked to ensure that all updating trials had longer RTs than control ones overall $(p<$ .05 for each comparison) consistent with the theoretical framework. The three probes (positive, intrusion, negative) were considered and analyzed separately.

The procedure replicated that of Experiment 1 exactly. Participants were randomly assigned to one of the two instruction groups and tested individually. Afterward, each participant was presented with a practice block of eight trials to familiarize them with the task. Two hundred and forty trials were then presented, shared equally in four blocks. About half of the trials presented the sublexical units in position 1-2 in the trigram, and the other half in position 2-3. We recorded subject-paced RT at each of the four phases as well as probe recognition accuracy and RT at Phase 5.

\section{Results}

\section{Accuracy and data treatment}

Participants performed accurately on an average of $93.80 \%$ of trials. We ran an ANOVA, with strength (weak-to-weak, weak-to-strong, strong-to-weak, strong-to-strong) as a within-participants factor, and instruction (disjunction, grouping) as a between-participants factor on mean accuracy rates of positive, intrusion, and negative responses. No effect reached significance $(p>.05)$. Only subject-paced RTs for trials that ended with correct probe recognition were analyzed. Trials with RTs of less than $150 \mathrm{~ms}$, or exceeding a participant's mean RT for each condition by more than three intraindividual standard deviations, were considered outliers and therefore excluded from further analyses (3.70\%).

\section{RT analyses}

An initial omnibus analysis was conducted to control for possible effects of position for sublexical units within the trigram. An ANOVA, with strength (weak-to-weak, weak-to-strong, strong-to-weak, strong-to-strong), phase (encoding, updating), and position (1-2, 2-3) as within-participant factors, and instruction (disjunction, grouping) as a between-participant factor, was conducted on subject-paced processing RTs.

Results showed no main effect of position $(p>.05)$. Moreover, position did not interact with strength $(p>.05)$.

After controlling for position effects (i.e., verifying these did not interact with the task), we collapsed positions (1-2) and (2-3) in order to run the following ANOVA, with strength, phase, and instruction on subject-paced RTs. A main effect of instruction reached significance, $F(1,48)=26.84, \eta_{\mathrm{p}}{ }^{2}=.36, p$ $<.001$, showing shorter RTs when the disjunction strategy was instructed. 


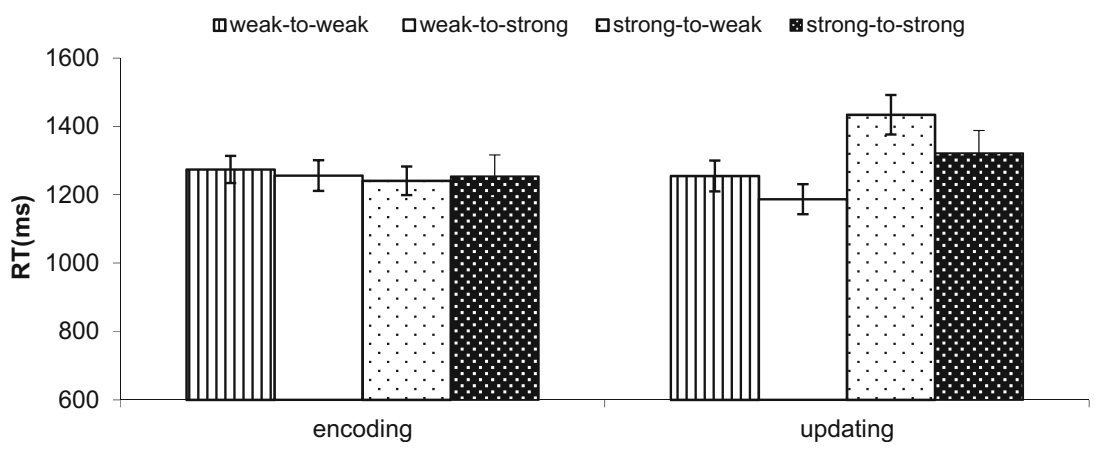

Fig. 4 Experiment 2: Mean RTs for each association strength as a function of phase (encoding, updating)

In addition, a main effect of strength reached significance, $F(3,46)=33.17, \eta_{p}^{2}=.69, p<.001$. We found that, on average, strong-to-weak associations had longer RTs, followed by the strong-to-strong associations; weak-to-strong associations, and the weak-to-weak associations. A main effect of Phase did not reach significance $(F<1)$.

The two-way interaction between phase and strength reached significance, $F(3,46)=20.42, \eta_{\mathrm{p}}^{2}=.57, p<.001$. Further analyses (planned comparisons, using Tukey's method) showed no significant differences between associations at the encoding phase $(F<1)$.

In contrast, at updating, all means were significantly different $(p<.001)$; strong associations needed longer RTs to be updated with weak association $(1,434 \mathrm{~ms})$, followed by strong associations to be updated with strong $(1,322 \mathrm{~ms})$, weak associations updated with weak $(1,255 \mathrm{~ms})$, and weak associations updated with strong $(1,187 \mathrm{~ms})$. See Fig. 4 . No other effect reached significance $(p>.05)$.

\section{Probe analysis}

Positive probes An analysis with strength (weak-to-weak, weak-to-strong, strong-to-weak, strong-to-strong) as a within-participant factor and instruction (disjunction, grouping) as a between-participant factor was conducted on correctly recognized positive probes. A main effect of instruction reached significance, $F(1,48)=6.69, \eta_{\mathrm{p}}^{2}=.12, p=.013$, showing shorter RTs when the disjunction strategy was instructed. No other effect reached significance $(p>.05)$.

Intrusion probes First, we conducted an analysis with strength (weak-to-weak, weak-to-strong, strong-to-weak, strong-to-strong) and probe (intrusion, negative) as withinparticipant factors and instruction (disjunction, grouping) as a between-participant factor, for intrusion probe versus negative probe RTs. We found a main effect of probe, $F(1,48)=$ $15.01, \eta_{\mathrm{p}}{ }^{2}=.24, p<.001$, showing longer RTs to recognize and respond to intrusion probes.

Afterward, to test our hypotheses more specifically, we ran an analysis on intrusions only (with strength and instruction as factors), as these represent a measure of the ability to control for no-longer-relevant information. Thus, these are needed to observe potential effects of the prolonged activation of strongly/weakly associated information. We found a main effect of instruction, $F(1,48)=4.40, \eta_{\mathrm{p}}^{2}=.084, p=.041$, showing, on average, shorter RTs when a disjunction strategy $(1,281 \mathrm{~ms})$ was instructed (relative to a grouping one, 1,471 $\mathrm{ms})$.

In addition, we found a main effect of strength, $F(3,45)=$ $13.14, \eta_{\mathrm{p}}^{2}=.46, p<.001$. Significantly longer RTs $(p<.001)$ were shown for recognition of an intrusion in strong-to-weak associations (1,467 ms), compared to weak-to-weak (1,330 $\mathrm{ms})$, strong-to-strong (1,325 ms) and weak-to-strong associations $(1,317 \mathrm{~ms})$. These latter three did not differ from each other $(p>.05)$. Instruction did not affect the association strength RTs $(p>.05)$.

\section{Discussion}

In Experiment 2, we replicated Experiment 1 in a more fully controlled design. Indeed, we were able to analyze recognition RTs, which were informative in testing our hypotheses. We also included a strong-to-strong association condition and controlled for position effects, which were shown not to affect the task. Longer RTs were shown to update strong association with weak, and more interestingly, we also showed the reverse result. That is, a weak association, updated with a strong one, resulted in shorter RTs. The effect is particularly interesting as it is observable for processing RTs (i.e., Experiment 1) but also for recognition RTs, with longer latencies for recognition (and interference control) from an item with a strong LTM association. In particular, we showed that interference control was greater when the intruding item belonged to an initial strong association; this, in turn, indicates the pervasiveness of the preexisting strong LTM association; one that is more activated and intrusive. As in Experiment 1, the disjunction strategy consistently favored updating, but never interacted with association strength. 


\section{General discussion}

Our aim was to investigate how sublexical LTM associations affect memory updating. Although such associations have been studied extensively in simple WM tasks utilizing recall (see, e.g., Gathercole et al., 1999), this is not the case with updating tasks. Different than recall, updating entails the active maintenance of goal-relevant information as well as the substitution of no-longer-relevant information.

Typically, the literature shows a boosting effect for recall; the stronger the preexisting association is in LTM, the better the performance is in WM. For updating, we showed the opposite; the stronger the preexisting association, the harder it is to dismantle it (i.e., longer RTs). In addition, when a strong association has to be reconstructed, this is usually enhanced (as shown by shorter RTs from weak to strong association). The result was observed both for processing speed (Experiments 1 and 2) and also when controlling interference from a previously activated strong association (Experiment 2).

Initially, we have to note that slowing of RTs in updating (i.e., relative to recall) could be related to the number of cognitive operations required in the two tasks. In fact, recall mainly involves maintenance of information; updating, in addition, embeds an item-substitution component. Therefore, it is reasonable to assume that an additional operation will also add a behavioral cost.

The current results appear inconsistent with findings that have studied the substitution component of updating specifically. For example, Lendínez et al. $(2011,2014)$ showed that similarity effects in the substitution process are beneficial to updating. However, it should be noted that they focused on the relation between old and new information. Instead, here, we considered the relation between items at encoding that are subsequently updated in WM. Thus, it is important to establish that these two perspectives differ in theoretical focus as well as analyses. Both relations examined have an effect on RT, but potentially, the underlying mechanisms are distinct. For Lendínez and colleagues (e.g., 2011), a hypothesized mechanism would consider substitution of nonshared features with information involved in updating (see also Nairne, 1990). The mechanism more likely to account for the present results is dismantling of previous associations, and strengthening of new associations between items maintained simultaneously (see, e.g., Schmiedek et al., 2009).

Thus, we believe that features models do not account for our results; instead, others, which consider the role of bindings build-up and dismantling are more consistent with our findings (e.g., Oberauer, 2005; Schmiedek et al., 2009). Specifically, here, we show how this continuous process of creation/dismantling of bindings during updating can be modulated by the strength with which the items are linked. Further, it is important to consider that Lendínez and colleagues manipulated similarity between digits, that is, a different cognitive domain to letter stimuli. In fact, letters and digits belong to two distinct systems, whose separation is well known, from behavioral, developmental, and neuropsychological studies (see, e.g., Szucs, Devine, Soltesz, Nobes, \& Gabriel, 2013).

In these current experiments, we explicitly instructed participants to use a strategy of item disjunction or item grouping. First, we observed that strategy induction can modulate task performance. In fact, when participants were not instructed to use a strategy (control group, Experiment 1), they were likely to use the grouping one, which is less functional to the task (as it produces longer RTs). When they were instructed to disjoint the items, their performance was enhanced. Thus, for the first time in the literature, the effect of an explicit strategy induction (and its beneficial effect) has been demonstrated in updating. Specifically, when participants kept items separated, working against spontaneous/natural binding, they were faster in dismantling associations and substituting irrelevant items with new ones. This was not the case when a different strategy (e.g., grouping) was applied.

We have to note, however, that although our results seem to suggest that instruction worked effectively, we cannot be sure our participants followed the instruction. Thus, we anticipate introducing a more controlled procedure for future studies (e.g., short interviews of our participants), in order to analyze the strategies used and the regulation of strategies through the materials used.

Accordingly, such beneficial effect for the disjunction strategy seems consistent with (and supportive of) the updating decomposition model proposed by Ecker et al. (2010). Further, it is reasonable to assume that, in order to substitute an item in WM (i.e., to update it), it would be helpful to anticipate its substitution by keeping each single item distinct rather than joining it with other items in a compound memory representation. The strategy of reducing association between items would then facilitate the updating process. In fact, disjunction would reduce the strength of associations and prepare for the updating process.

Further, we believe this point allows explanation of the absence of interaction between instruction and association strength. If we take into account the preemptive intervention of the disjunction strategy, we see how items might remain unbound when they are encoded (and thus are coded as single memory units), independently of the kind of LTM association (i.e., strong or weak) with which they were activated. In addition, we acknowledge that the sublexical stimuli we administered could be too implicit/abstract and produce a modest modulation based on their explicitly controlled associative strength. For this reason, future studies could consider more explicit/overt material and related manipulations, such as words or categorical associations.

Our data clearly demonstrate that associations/bindings are crucial in the updating process. In fact, these results 
demonstrate that on the one hand, strong associations are dismantled/updated with greater difficulty (i.e., they need longer RTs), and on the other, strong item associations are reinforced with less difficulty (shorter RTs). From this evidence, then, we could argue that the nature of updating rests in the interplay between breaking up and rebuilding bindings through different memory systems such as WM (see Artuso \& Palladino, 2016; Schmiedek et al., 2009) and recollective episodic LTM (see Boujut \& Clarys, 2015).

Therefore, if we assume that item substitution in LTM associations is the distinctive updating component (in line with Ecker et al., 2010), we believe it is also important to examine how strongly the specific item is linked to others retained in LTM. In fact, the quality of the association between stimuli is likely to modulate the substitution of one of them, enhancing or interfering with the substitution process.

In summary, we found a cost for dismantling a strong preexisting association from LTM (see longer RTs for strong-to-weak and strong-to-strong conditions), and a benefit in build-up of a strong preexisting association (see shorter RT for weak-to-weak and weak-to-strong conditions). We believe our findings represent an original and innovative contribution to literature. They suggest conceptualization of the updating process as sensitive to the nature of the to-be-updated material, and to both the LTM system contents and task-strategic approach. Specifically, this would indicate updating to be affected by the nature of the information and, more importantly here, by the strength of association between information. In addition, we have showed that effects of explicit instruction in approach to an updating task are evident during updating. Future research should focus on how more complex memory material and different types of bindings may interact with the updating process and potentially affect its efficiency.

Acknowledgements We wish to thank Candice Morey, Santiago Pelegrina, and a third reviewer for their helpful comments and suggestions, and Sara Bottani for her help with data collection.

\section{References}

Acheson, D. J., \& MacDonald, M. C. (2009), Verbal working memory and language production: Common approaches to the serial ordering of verbal information. Psychological Bulletin, 135, 50-68. doi: https://doi.org/10.1037/a0014411

Artuso, C., Cavallini, E., Bottiroli, S., \& Palladino, P. (2017). Updating working memory: Memory load matters with aging. Aging Clinical and Experimental Research, 29, 371-377. doi:https://doi.org/10. 1007/s40520-016-0581-y

Artuso, C., \& Palladino, P. (2011). Content-context binding in verbal working memory updating: On-line and off-line effects. Acta Psychologica, 136, 363-369. doi:https://doi.org/10.1016/j.actpsy. 2011.01.001

Artuso, C., \& Palladino, P. (2014). Binding and content updating in working memory tasks. British Journal of Psychology, 105, 226242. doi:https://doi.org/10.1111/bjop.12024.
Artuso, C., \& Palladino, P. (2016). Modulation of working memory updating: Does long-term memory lexical association matter? Cognitive Processing, 17, 4957. doi:https://doi.org/10.1007/ s10339-015-0735-4

Artuso, C., Palladino, P., \& Ricciardelli, P. (2015). Social updating: The role of gaze direction in updating and memorizing emotional faces. Social Cognition, 33, 543-561. doi:https://doi.org/10.1521/soco. 2015.33.6.543

Boujut, A., \& Clarys, D. (2015). The effect of ageing on recollection: The role of the binding updating process. Memory, 24, 1231-1242. doi: https://doi.org/10.1080/09658211.2015.1091893

Bunting, M., Cowan, N., \& Saults, J. S. (2006). How does running memory span work? The Quarterly Journal of Experimental Psychology, 59, 1691-1700. doi:https://doi.org/10.1080/17470210600848402

Chein, J. M., \& Morrison, A. B. (2010). Expanding the mind's workspace: Training and transfer effects with a complex working memory span task. Psychonomic Bulletin \& Review, 17, 193-199. doi:https://doi.org/10.3758/PBR.17.2.193

Cowan, N. (2001). The magical number 4 in short-term memory: A reconsideration of mental storage capacity. Behavioral Brain Sciences, 24, 87-114.

Cowan, N. (2017). Mental objects in working memory: Development of basic capacity or of cognitive completion? Advances in Child Development and Behavior, 52, 81-104. doi:https://doi.org/10. 1016/bs.acdb.2016.12.001

Daneman, M., \& Carpenter, P. A. (1980). Individual differences in working memory and reading. Journal of Verbal Learning and Verbal Behavior, 19, 450-466. doi:https://doi.org/10.1016/S0022-5371(80) 90312-6

De Mauro, T., Mancini, F., Vedovelli, M., \& Voghera, M. (1993). Lessico di frequenza dellitaliano parlato. [Frequency lexicon of spoken Italian language]. Milan, Italy: Etas Libri.

Ecker, U. C., Lewandowsky, S., Oberauer, K., \& Chee, A. E. (2010). The components of working memory updating: An experimental decomposition and individual differences. Journal of Experimental Psychology: Learning, Memory, and Cognition, 36, 170-189. doi: https://doi.org/10.1037/a0017891

Gathercole, S. E., Frankish, C. R., Pickering, S. J., \& Peaker, S. (1999). Phonotactic influences on short-term memory. Journal of Experimental Psychology: Learning, Memory, and Cognition, 25, 84-95. doi:https://doi.org/10.1037/0278-7393.25.1.84

Hulme, C., Stuart, G., Brown, G. D. A., \& Morin, C. (2003). High- and low-frequency words are recalled equally well in alternating lists: Evidence for associative effects in serial recall. Journal of Memory and Language, 49, 500-518. doi:https://doi.org/10.1016/S0749596X(03)00096-2

Lendínez, C., Pelegrina, S., \& Lechuga, M. T. (2011). The distance effect in numerical-memory updating task. Memory \& Cognition, 39, 675685. doi:https://doi.org/10.3758/s13421-010-0047-y

Lendínez, C., Pelegrina, S., \& Lechuga, M. T. (2014). The role of similarity in updating numerical information in working memory: Decomposing the numerical distance effect. Quarterly Journal of Experimental Psychology, 67, 16-32. doi:https://doi.org/10.1080/ 17470218.2013.793375

Majerus, S. (2013). Language repetition and short-term memory: An integrative framework. Frontiers in Human Neuroscience, 7(357). doi:https://doi.org/10.3389/fnhum.2013.00357

Majerus, S., Perez, T. M., \& Oberauer, K. (2012). Two distinct origins of long-term learning effects in verbal short-term memory. Journal of Memory and Language, 66, 38-51. doi:https://doi.org/10.1016/j. jm1.2011.07.006

Majerus, S., Van der Linden, M., Mulder, L., Meulemans, T., \& Peters, F. (2004). Verbal short-term memory reflects the sublexical organization of the phonological language network: Evidence from an incidental phonotactic learning paradigm. Journal of Memory and Language, 51, 297-306. doi:https://doi.org/10.1016/j.jml.2004.05.002 
Martin, N., \& Saffran, E. M. (1992), A computational account of deep dysphasia: Evidence from a single case study. Brain and Language, 43, 240-274. doi:https://doi.org/10.1016/0093-934X(92)90130-7

McNamara, D. S., \& Scott, J. L. (2001). Working memory capacity and strategy use. Memory \& Cognition, 29, 10-17. doi:https://doi.org/ 10.3758/BF03195736

Morris, N., \& Jones, D. M. (1990). Memory updating in working memory: The role of central executive. British Journal of Psychology, 81, 111-121. doi:https://doi.org/10.1111/j.2044-8295.1990.tb02349.x

Morrison, A. B., \& Chein, J. M. (2011). Does working memory training work? The promise and challenges of enhancing cognition by training working memory. Psychonomic Bulletin and Review, 18, 46-60. doi:https://doi.org/10.3758/s13423-010-0034-0

Morrison, A. B., Rosenbaum, G. M., Fair, D., \& Chein, J. M. (2016). Variation in strategy use across measures of verbal working memory. Memory \& Cognition, 9, 922-936. doi:https://doi.org/10.3758/ s13421-016-0608-9

Nairne, J. S. (1990). A feature model of immediate memory. Memory \& Cognition, 18, 251-269. doi:https://doi.org/10.3758/BF03213879

Oberauer, K. (2005). Binding and inhibition in working memory: Individual and age differences in short-term recognition. Journal of Experimental Psychology: General, 134, 368-387. doi:https:// doi.org/10.1037/0096-3445.134.3.368

Palladino, P., Cornoldi, C., De Beni, R., \& Pazzaglia, F. (2001). Working memory and updating processes in reading comprehension. Memory \& Cognition, 29, 344-354. doi:https://doi.org/10.3758/ BF03194929
Palladino, P., \& Jarrold, C. (2008). Do updating tasks involve updating? Evidence from comparisons with immediate serial recall. The Quarterly Journal of Experimental Psychology, 61, 392-399. doi: https://doi.org/10.1080/17470210701664989

Poirier, M., \& Saint-Aubin, J. (2005). Word frequency effects in immediate serial recall: Item familiarity and item co-occurrence have the same effect. Memory, 13, 325-332. doi:https://doi.org/10.1080/ 09658210344000369

Saint-Aubin, J., Ouellette, D., \& Poirier, M. (2005). Semantic similarity and immediate serial recall: Is there an effect on all trials? Psychonomic Bulletin and Review, 12, 171-177. doi:https://doi. org/10.3758/BF03196364

Schmiedek, F., Hildebrandt, A., Lövdén, M., Wilhelm, O., \& Lindenberger, U. (2009). Complex span versus updating tasks of working memory: The gap is not that deep. Journal of Experimental Psychology: Learning, Memory, and Cognition, 35, 1089-1096. doi:https://doi.org/10.1037/a0015730

Szucs, D., Devine, A., Soltesz, F., Nobes, A., \& Gabriel, F. (2013). Developmental dyscalculia is related to visuo-spatial memory and inhibition impairment. Cortex, 49, 2674-2688. doi:https://doi.org/ 10.1016/j.cortex.2013.06.007

Thorn, A., \& Frankish, C. (2005). Long-term knowledge effects on serial recall of nonwords are not exclusively lexical. Journal of Experimental Psychology: Learning, Memory, and Cognition, 31, 729-735. doi:https://doi.org/10.1037/0278-7393.31.4.729 